\title{
Abstract: Populationsbasierte 4D Bewegungsatlanten für VR Simulationen
}

\author{
Andre Mastmeyer, Matthias Wilms, Heinz Handels \\ Institut für Medizinische Informatik, Universität zu Lübeck \\ mastmeyer@imi.uni-luebeck.de
}

Atembewegte Avatare können in einem kürzlich vorgestellten visuo-haptischen Virtual Reality (VR) 4D-Simulatorkonzept modelliert [1] und GPU-basiert dargestellt [2] werden. Nadelinterventionsimulationen im hepatischen Bereich mit atmenden virtuellen Patientenkörpern sind aktuell ohne die patientenspezifische, dosisrelevante 4D-Datenerfassung nicht durchführbar. Hierbei kann ein populationsbasierter Ansatz zur Modellierung eines gemittelten, übertragbaren (4DAtembewegungsatlas) abhelfen und die Risiken einer dosisrelevanten und teuren Erfassung eines 4D-Datensatzes mindern [3]. Diese Modelle können durch nichtlineare Registrierung auf den statischen und zu animierenden 3D-CT-Datensatz eines neuen Patienten übertragen werden [3].

Die Atmungsmodellierung mit einem linearen Vektorfeldregressionsmodell basiert auf einer Menge von 4D-CT-Datensätzen mit 3D-Atemphasenbildern und einem Spirometriesignal mit einer Hysteresesignalkomponente. IntrapatientInterphasen-Bildregistrierungen zu einer ausgewählten patientenspezifischen Referenzphase (z.B. der maximalen Einatmung) bilden den ersten Arbeitspunkt eines Dreischritts, der in einem personalisierten Atemmodell resultiert.

Der nächste Schritt ist die Mittelung der personalisierten Atmungsmodelle. Für einen gemeinsamen Referenzrahmen wird die Atemphase bspw. der maximalen Einatmung eines Referenzpatienten ausgewählt. Dieser Rahmen ist das Ziel von Interpatientenregistrierungen der gewählten Referenzphasen. Mit den in den gleichen gemeinsamen Bezugsrahmen transformierten Bewegungsinformationen ergibt ein Mittelungsprozess das Gruppenintensitäts- und -bewegungsmodell.

Schliesslich wird das mittlere 4D-Patientenatmungsmodells durch eine personalisierende Interpatientenregistrierung auf neue 3D-Patientendaten transformiert. Diese müssen lediglich dosis- und kostensparend als 3D-CT-Datensatz in Referenzatemlage aufgenommen worden sein und können im 4D-VR-Simulator visuo-haptisch animiert [2] werden ${ }^{1}$.

\section{Literaturverzeichnis}

1. Mastmeyer A, et al. Efficient patient modeling for visuo-haptic VR simulation using a generic patient atlas. Comp Meth Prog Biomed. 2016;132:161-175.

2. Fortmeier D, et al. Direct visuo-haptic $4 \mathrm{D}$ volume rendering using respiratory motion models. IEEE Trans Haptics. 2015;8(4):371-383.

3. Mastmeyer A, et al. Population-based respiratory 4D motion atlas construction and its application for VR simulations of liver punctures. Proc SPIE. 2018; p. accepted.

\footnotetext{
${ }^{1}$ VR-Simulation: 4D Intensitäts- und Atembewegungsatlas: https://goo.gl/Qog138
} 